\title{
Cabergoline Induced Supraventricular Tachycardia with QT Prolongation
}

\author{
Vineetha Bharathan Menon ${ }^{1 *}$, Bhanuvaishnavi Gaddipati ${ }^{2}$, Venkatesh $\mathbf{C ~ R}^{3}$, Pratibha Pereira ${ }^{4}$, Hathur \\ Basavanna Gowdappa ${ }^{5}$, Madhan Ramesh ${ }^{6}$
}

${ }^{1}$ Doctoral Research Scholar, Department of Clinical Pharmacy, JSS Medical College and Hospital, JSS University, Mysore, Karnataka, INDIA

${ }^{2}$ PharmD Intern, Department of Clinical Pharmacy, JSS Medical College and Hospital, JSS University, Mysore, Karnataka, INDIA ${ }^{3}$ Senior Resident, Department of General Medicine, JSS Medical College and Hospital, JSS University, Mysore, Karnataka, INDIA ${ }^{4}$ Associate Professor, Department of General Medicine, JSS Medical College and Hospital, JSS University, Mysore, Karnataka, INDIA ${ }^{5}$ Professor and Dean, Department of General Medicine, JSS Medical College and Hospital, JSS University, Mysore, Karnataka, INDIA ${ }^{6}$ Professor and Head, Department of Clinical Pharmacy, JSS Medical College and Hospital, JSS University, Mysore, Karnataka, INDIA

\begin{abstract}
Background: Cabergoline is commonly known to cause valvular disorders. Cases of supraventricular tachycardia with QT prolongation are seen, albeit, extremely rare. Aim: We report a case of supraventricular tachycardia with QT prolongation presumed secondary to cabergoline use. Clinical Details: A 30-year-old post-partal female complained of palpitation and syncope after two days of cabergoline usage. Her past medical history was insignificant. Electrocardiogram showed QT prolongation of $489 \mathrm{~ms}$. Based on her presentation, we presume that the patient's condition was the result of cabergoline. Outcomes: Causality assessment was suggestive of a probable relationship between the patient's symptoms and her use of cabergoline. Symptoms improved significantly after the offending drug was withdrawn with an objective reduction in QTc. Conclusion: Electrocardiogram should be monitored in individuals receiving more than one QT prolonging medication or with symptoms of arrhythmia or are at high risk of QT prolongation/Torsades de Pointes.
\end{abstract}

Key words: Adverse Effects, Arrhythmia, Cabergoline, QT Prolongation, Supraventricular Tachycardia, Torsades de Pointes.

\section{INTRODUCTION}

Cabergoline is primarily used for the treatment of galactorrhoea, prolactinomas and Parkinson's disease. ${ }^{1,2}$ Adverse effects associated with cabergoline are usually mild to moderate and include nausea, constipation, dry mouth, gastric irritation, dyspepsia, sleep disturbances, vertigo, depression, dyskinesia, hallucinations, hypotension, peripheral edema, arrhythmia and angina pectoris. ${ }^{2}$ Herein we present a case of supraventricular tachycardia with QT prolongation which was presumably caused by cabergoline.

\section{CASE REPORT}

A 30-year-old female, eight weeks postpartum, with a BMI of $13.0 \mathrm{~kg} / \mathrm{m}^{2}$ was admitted to a tertiary care hospital with history of loose stools, 3-4 episodes/ day, since three days. She complained of generalized weakness and loss of appetite since two weeks. Her past medical history was insignificant. She had an uncomplicated C-Section with delivery of a healthy baby eight weeks ago. On examination, her blood pressure (BP) and heart rate $(\mathrm{HR})$ were $104 / 70 \mathrm{mmHg}$ and $86 \mathrm{bpm}$, respectively. Physical examination revealed pallor. However, other systems were clinically unremarkable. Based on her complaints, a clinical diagnosis of anemia was made. Relevant investigations to evaluate anemia were advised and based on her complete blood count $(\mathrm{CBC})$ test reports and peripheral
DOI: 10.5530/ijopp.10.2.30

Address for correspondence: Dr. Vineetha Bharathan Menon

Research Scholar, Department of Clinical Pharmacy, JSS Hospital, Mysore, Karnataka, INDIA. Phone no: 9538283470

E-mail: vineethamenon54@ yahoo.com 
blood smear findings (Hb: $3.2 \mathrm{gm} / \mathrm{dl}$, PCV: $9.7 \%$, RBC: 1.22 million/cumm, MCV: 79.5 fl, MCH: 26.2 pg, MCHC: $33.0 \mathrm{gm} / \mathrm{dl}$, RDW: $24.9 \%$, TIBC: 131 $\mu \mathrm{g} / \mathrm{dl}$, TLC: 2920 cells/cumm, PLT: 38000 lakhs/ cumm), a diagnosis of pancytopenia was made and evaluated with bone marrow aspiration and biopsy, which revealed erythroid and megakaryocytic hyperplasia. Her other tests were normal.

Following admission, patient was treated with packed red blood cell (PRBC) transfusion and ceftriaxone $1 \mathrm{gm}$ twice daily. On day two, she complained of mastalgia for which she was referred to the obstetrics and gynecology department. There she was found to have right breast engorgement and was advised cabergoline $0.5 \mathrm{mg}$ twice daily for lactation suppression. On the fourth day, patient complained of palpitation, profuse sweating and giddiness. Considering transfusion reactions, PRBC was stopped. On the fifth day of hospitalization, patient again complained of palpitations and syncope. Electrocardiogram (ECG) done at this juncture showed QT prolongation of $400 \mathrm{~ms}$ (Bazzett's) with supraventricular tachycardia (Figure 1). On the sixth day, patient complained of gastric irritation along with palpitation and syncope and had an episode of supraventricular tachycardia with QT prolongation. In the view of her gastric irritation, ceftriaxone was stopped and she was started on esomeprazole 40 mg once daily and magaldrate 2 tablespoon thrice a day. Next day, as her complaints were persisting, ECG was repeated which revealed inverted $T$ wave in V1 and V5 lead with QT prolongation of $489 \mathrm{~ms}$ (Bazzett's) (Figure 2). Troponin and and creatine kinase-MB isoenzyme levels were $0.027 \mathrm{mcg} / \mathrm{L}$ and 1.27 IU/L respectively. Her hematological parameters repeated at this juncture showed improvement of all the three cell lines (Hb: $9.7 \mathrm{gm} / \mathrm{dl}, \mathrm{PCV}: 27.1$ \%, RBC: 2.91 million/cumm, MCV: 93.1f, MCH: 29.2 pg, MCHC: 34.4 gm/dl, RDW: $22.0 \%$, TLC: 9290 cells/cumm, PLT: 3.16 lakhs/cumm).

On the eight day, patient had the same complaints of palpitation and syncope and her HR was $140 \mathrm{bpm}$, missed beat + , regularly irregular and BP was 100/70 mmHg. ECG showed ventricular bigemy and and echocardiogram report was was within normal limits. Impression was made as probable drug induced

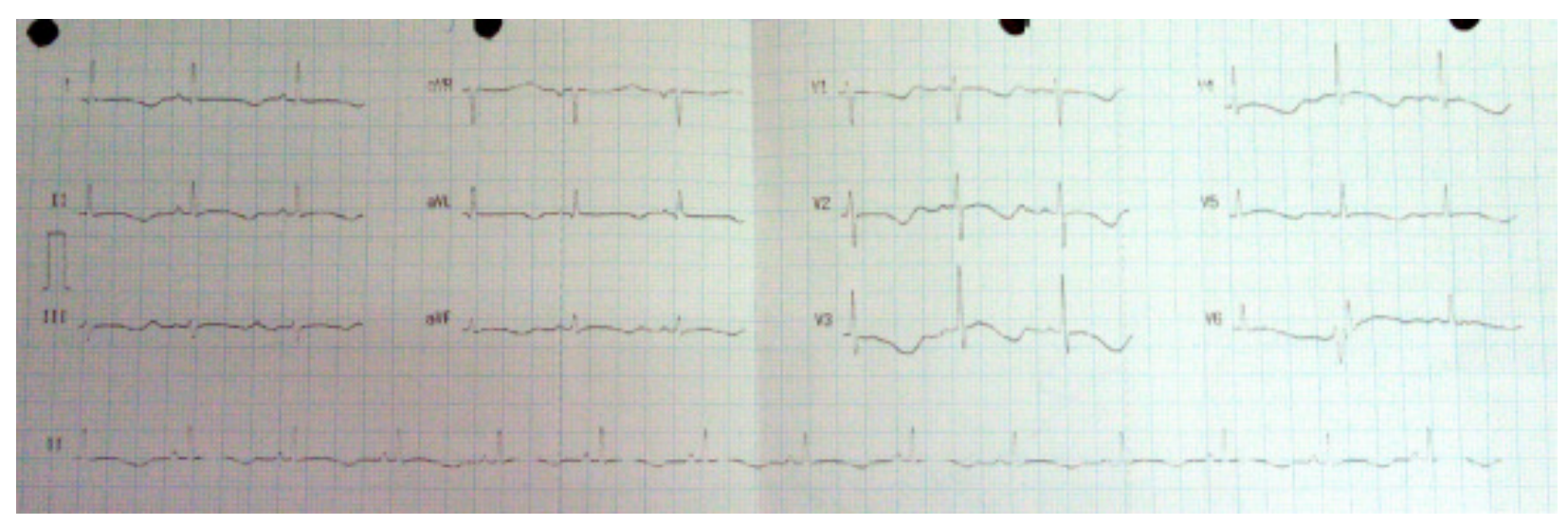

Figure 1: ECG showing QT prolongation with global T inversions.

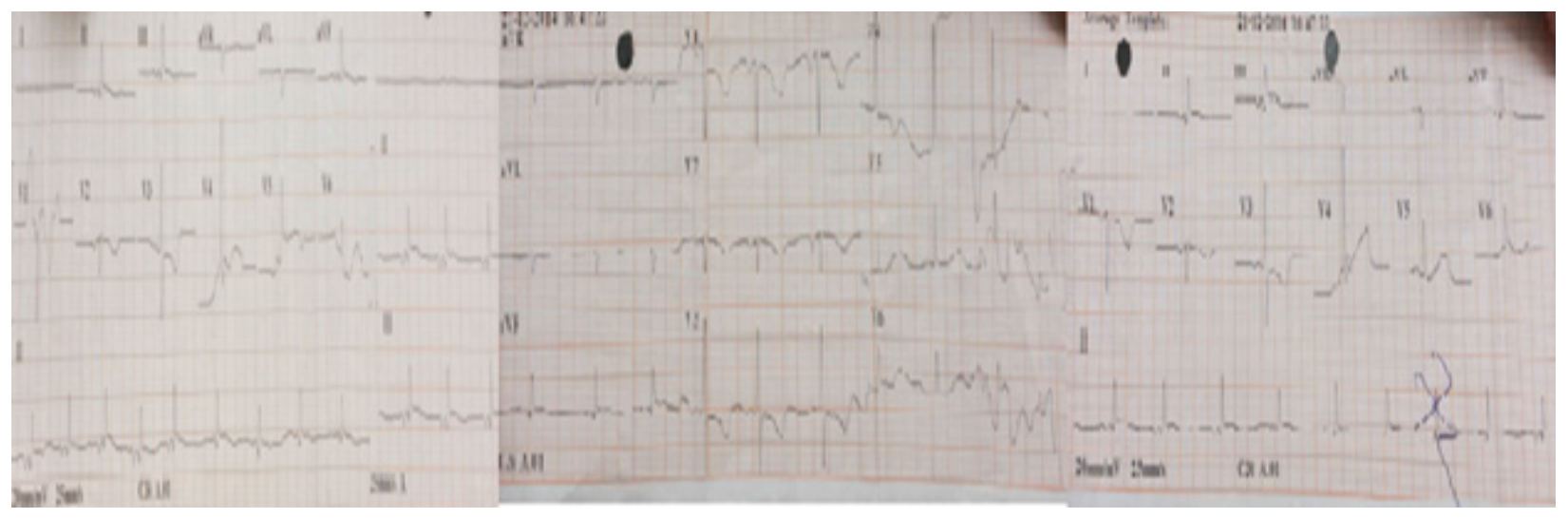

Figure 2: Patient's ECG during the episode of supraventricular tachycardia (AV nodal re-entry tachycardia). 
arrhythmia, paroxysmal supraventricular tachycardia with QT prolongation. Esomeprazole and cabergoline were put on hold and metoprolol $25 \mathrm{mg}$ once daily, furosemide $40 \mathrm{mg}$ /amiloride $5 \mathrm{mg}$ twice daily and amiodarone $100 \mathrm{mg}$ twice daily were started. On day nine, arrhythmia persisted and patient continued to have palpitation and a syncope, had a fall and suffered minor injury over right side of her scalp. Her medications, amiodarone and furosemide/ amiloride, except magaldrate and metoprolol were withdrawn. The next day patient again had QT prolongation with palpitation. She was then referred to a cardiologist, for which he advised holter monitoring and withdrawal of $\beta$ blocker. However, as there were no further complaints over the next few days and ECG normalized, holter ECG was deferred and the patient was discharged. In view of the patient's typical clinical presentation, diagnosis of cabergoline induced supraventricular tachycardia with QT prolongation was made. Post discharge, she was followed up over a period of one month and reported no further episodes of palpitations.

The Naranjo Nomogram for Adverse Drug Reaction Assessment and WHO-UMC Causality Assessment System were applied to determine the causality for suspected adverse drug reaction (ADR). The causality assessment with both scales revealed that the ADR due to cabergoline in this case was probable (Naranjo overall score: 7). Severity of the ADR was evaluated using the Modified Hartwig and Siegel scale, based on which it was categorized as moderate (level 4(a)) reaction.

\section{DISCUSSION}

Acquired forms of QT prolongation and arrhythmia, particularly related to drug therapy, are mainly due to drug effects on the ion channels responsible for the cardiac action potential, mostly via reduction in the outward potassium current, either by inhibition of IKr or IKs. ${ }^{3}$ Excessive QT prolongation can lead to the development of early after-depolarisations that can then trigger severe polymorphic ventricular tachycardia or Torsades de Pointes (TdP). If $\mathrm{TdP}$ is rapid or prolonged, it can lead to ventricular fibrillation or arrhythmia. ${ }^{4,5}$

While studies have identified association between long-term treatment with cabergoline and cardiac valve abnormalities, including tricuspid regurgitation, mitral regurgitation and aortic regurgitation, ${ }^{1}$ cabergoline induced arrhythmia is very rare and are reported in less than $1 \%$ patients,${ }^{6}$ particularly supraventricular tachycardia with QT prolongation for which only 2 cases has been reported in VigiAccess. In addition to the above mentioned mechanisms, the activation of $\alpha 1$ - and $\alpha 2$-adrenoceptors and DA1 and DA2 dopamine receptors by cabergoline may also play an important role in the pathophysiology of arrhythmia. There is an extreme individual variation in the amount of dopamine required to activate different receptors. DA1 and DA2 receptors are activated at the lowest dose resulting in a decline in peripheral vascular resistance and modest increments in renal blood flow, urine volume and sodium excretion. As the dose is increased, $\beta$ 1-adrenoceptors are activated and cardiac output begins to increase, typically with little change in HR. At higher doses recruitment of $\alpha 1$ - and $\alpha 2$-adrenoceptors occurs resulting in increase in vascular resistance or until excessive $\beta$ 1-adrenoceptor activity leads to tachycardia or ventricular arrhythmias. ${ }^{7}$ Genetic factors could also had played an important role in our patient. Research has demonstrated that an SCN5A promoter polymorphism common in Asians modulates the duration of the PR and QRS intervals, thereby altering the action potential. ${ }^{3}$ Cabergoline is absorbed from the gastrointestinal tract within 0.5 to $4 \mathrm{~h}^{2}$ and has an average elimination half-life of 65 $\mathrm{h}$ that may provide continuous dopaminergic stimulation when administered once daily. ${ }^{8}$ Although the patient had delayed onset of symptoms, the long elimination half-life of cabergoline may contribute to its prolonged duration of action and persistence of symptoms even after drug withdrawal, as observed in our patient.

The duration of QT interval is the major determinant of the risk of drug-induced TdP. ${ }^{5} \mathrm{An}$ increase in the QTc by more than $60 \mathrm{~ms}$ is generally considered to confer a high risk of $\mathrm{TdP}^{9}$ and most cases of drug-induced arrhythmia result in prolongation of the QTc to values greater than $500 \mathrm{~ms}^{3}$ In our patient, the corrected QT interval was recorded as $400 \mathrm{~ms}$ and $489 \mathrm{~ms}$ (Bazzett's) in the first and second ECG respectively. Thus, although the patient was at high risk of $\mathrm{TdP}$, the duration of QTc did not correlate with the previously established values of druginduced arrhythmia. Congenital long QT syndrome, history of previous drug-induced QT prolongation, older age, female gender, structural heart disease, renal or hepatic disease, electrolyte abnormalities, absolute or relative bradycardia, use of more than one QT prolonging drug or metabolic inhibitors of another QT prolonging drugs, starvation or obesity also increase the risk of $\mathrm{TdP}$ and potentiate the QT prolonging effects of drugs., ${ }^{5,9}$ Other than being a female and having received multiple QT prolonging 
drugs, none of the other above mentioned risk factors were present in the patient.

Among the medications patient received, PRBC transfusion is known to cause palpitation ${ }^{10}$ and use of esomeprazole, furosemide, amiloride and amiodarone are reported to cause arrhythmias. ${ }^{11}$ However, she received esomeprazole, furosemide, amiloride and amiodarone after developing palpitations and thus can be ruled out as a possible cause of the arrhythmia. PRBC transfusions are known to cause acute febrile, non-hemolytic reactions like fever, chills, headache, flushing, palpitations, cough, chest tightness, increased HR and flank pain. ${ }^{10}$ Other than palpitation and tachycardia, our patient did not have any of the other above mentioned symptoms. Thus, based on the time temporal relationship for development of the event and clinical presentations, we contend that this clinical event of supraventricular tachycardia with QT prolongation was due to cabergoline.

\section{CONCLUSION}

Cardiac arrhythmias caused by non-cardiac drugs are a growing concern because most of these cases fall outside the doctor's expectation, which makes it difficult to take preventive and therapeutic measures. It is for this reason that it is important to identify drugs that are likely to cause QT prolongation. Prescribers should thus consider the possibility of drug-induced QT prolongation in patients presenting with syncope or palpitations after receiving cabergoline. Baseline and periodic ECGs should be performed in patients receiving more than one QT prolonging drug, with symptoms of arrhythmia or in patients at high risk of QT prolongation.

\section{ACKNOWLEDGEMENTS}

The authors would like to thank the Principal, JSS College of Pharmacy and JSS University, Mysore for the support and encouragement.

\section{CONFLICT OF INTEREST}

The authors declare that they have no conflicts of interest that are directly relevant to the content of the case report.

\section{ABBREVIATION USED}

ADRs: Adverse Drug Reactions; BP: Blood Pressure; CBC: Complete Blood Count; ECG: Electrocardiogram; HR: Heart Rate; PRBC: Packed Red Blood Cell; TdP: Torsades de Pointes.

\section{REFERENCES}

1. Rhee SS, Pearce EN. The endocrine system and the heart: a review. Revista Espa-ola de Cardiología (English Edition). 2011;64(3):220-31. https://doi. org/10.1016/j.rec.2010.10.016.

2. Razmjoo H, Rezaei L, Dehghani A, Peyman A, Akhlaghi M. Bilateral angleclosure glaucoma in a young female receiving cabergoline: a case report. Case reports in ophthalmology. 2011;2(1):30-3. https://doi.org/10.1159/000324099; PMid:21347189 PMCid:PMC3042015.

3. Heist EK, Ruskin JN. Drug-induced arrhythmia. Circulation. 2010;122(14):142635. https://doi.org/10.1161/CIRCULATIONAHA.109.894725; PMid:20921449.

4. Raj SR, Stein CM, Saavedra PJ, Roden DM. Cardiovascular effects of noncardiovascular drugs. Circulation. 2009;120(12):1123-32. https:// doi.org/10.1161/CIRCULATIONAHA.107.728576; PMid:19770411 PMCid:PMC2773827.

5. Cubeddu LX. QT prolongation and fatal arrhythmias: a review of clinical implications and effects of drugs. American journal of therapeutics. 2003;10(6):452-7. https://doi.org/10.1097/00045391-200311000-00013; PMid:14624285.

6. Cabergoline Monograph- BC Cancer Agency Cancer Drug Manual. http:// www.bccancer.bc.ca/drug-database-site/Drug\%20Index/Cabergoline_ monograph_1Sept09.pdf(accessed on 2015 Aug 17).

7. Goldberg LI, Rajfer SI. Dopamine receptors: applications in clinical cardiology. Circulation. 1985;72(2):245-8. https://doi.org/10.1161/01.CIR.72.2.245.

8. Rinne UK, Bracco F, Chouza C, et al. Cabergoline in the treatment of early parkinson's disease Results of the first year of treatment in a double-blind comparison of cabergoline and levodopa. Neurology. 1997;48(2):363-8. https:// doi.org/10.1212/WNL.48.2.363; PMid:9040722.

9. Drug-induced QT prolongation and Torsades de Pointes - the facts. Prescriber Update; 31: 27-29.

10. Blood Administration and Transfusion Reactions; c2005. http://www.austincc. edu/adnlev4/rnsg2331online/lab/641.pdf. (accessed on 2015 Aug 17).

11. McEvoy GK, ed in chief, Snow ED, ed. AHFS: Drug Information. Bethesda, MD: American Society of Health-System Pharmacists. 2013:2691-2. 